drives tumor progression by accelerating the rate of gain or loss of individual chromosomes. The molecular mechanisms of chromosomal instability in human cancers largely remain to be elucidated.

Several years ago, we hypothesized that mutations in human spindle checkpoint genes could cause chromosomal instability in cancers $^{11}$. We cloned the two human homologs of yeast $B u b 1$, called $B U B 1$ and $B U B 1 B$, and found that the two genes were mutated, albeit rarely, in chromosomal instability tumors of individuals with colorectal cancer ${ }^{2}$. This study provided the first evidence that gene mutations can result in a defective spindle checkpoint in humans and thus might be responsible for aneuploidy in human cancers. One $B U B 1 B$ mutation in an aneuploid colorectal cancer was a somatic deletion that was predicted to remove part of the protein's kinase domainanalogous to the truncating mutations now found in individuals with MVA.

Mutant mice that express low levels of BubR1 protein develop progressive aneuploidy but form tumors only after being challenged with a carcinogen ${ }^{12}$. These findings, as well as the low frequency of $B U B 1$ and $B U B 1 B$ mutations in cancers and the lack of gene mutations found in any other members of the mitotic checkpoint machinery thus far, led to questions regarding the importance and applicability of our original observations. The belief that spindle checkpoint defects cause aneuploidy in human tumors, however, is revived by pinning down $B U B 1 B$ mutations as the underlying genetic alteration in MVA.
1. Jallepalli, P.V. \& Lengauer, C. Nat. Rev. Cancer 1, 109-117 (2001).

2. Cahill, D.P. et al. Nature 392, 300-303 (1998).

3. Gemma, A. et al. Genes Chromosomes Cancer 29, 213-218 (2000).

4. Hanks, S. et al. Nat. Genet. 36, 1159-1161 (2004).

5. Vigfusson, N.V. et al. Cytogenet. Cell Genet. 42, 154-158 (1986)

6. Warburton, D., Anyane-Yeboa, K., Taterka, P., Yu, C.Y. \& Olsen, D. Ann. Genet. 34, 287-292 (1991).

7. Jacquemont, S., Boceno, M., Rival, J.M., Mechinaud, F. \& David, A. Am. J. Med. Genet. 109, 17-21 (2002).

8. Plaja, A. et al. Am. J. Med. Genet. 98, 216-223 (2001).

9. Matsuura, S. et al. Am. J. Hum. Genet. 67, 483-486 (2000)

10. Musacchio, A. \& Hardwick, K.G. Nat. Rev. Mol. Cell. Biol. 3, 731-741 (2002).

11. Lengauer, C., Kinzler, K.W. \& Vogelstein, B. Nature 396, 643-649 (1998).

12. Dai, W. et al. Cancer Res. 64, 440-445 (2004).

\title{
Complementing complexity
}

\author{
Trudy F C Mackay
}

One challenge in modern biology is to understand the detailed genetic basis of variation for quantitative traits, including complex behaviors. A new study shows that historical recombination in outbred strains combined with functional complementation tests can identify pleiotropic genes with small effects on naturally occurring variation for anxiety-related behaviors in mice.

\begin{abstract}
Most variation between individuals in behavior, physiology, morphology, disease susceptibility and reproductive fitness can be attributed to the segregation of multiple quantitative trait loci (QTLs) with individually small effects, whose expression is conditional on the environment ${ }^{1}$. Understanding the genetic and environmental factors that cause this variation is of fundamental importance for medicine, agriculture, evolution and the emerging discipline of functional genomics. Genome scans for QTLs have become a cottage industry in the past 15 years, facilitated by the discovery of abundant, highly polymorphic molecular markers in many species and the development of sophisticated statistical methods of analy$\mathrm{sis}^{2,3}$. Identifying the genes underlying QTL peaks by positional cloning has proven elusive in all but a handful of cases where the QTLs had larger than usual effects ${ }^{4}$. On page 1197

Trudy F. C. Mackay is in the Department of Genetics, Campus Box 7614, North Carolina State University, Raleigh, North Carolina 27612, USA.

e-mail:trudy_mackay@ncsu.edu
\end{abstract}

of this issue, Binnaz Yalcin and colleagues ${ }^{5}$ describe a combined genetic and functional strategy for identifying genes corresponding to QTLs with small effects.

\section{The elusive QTLs}

There are three main problems facing highresolution QTL mapping. First, an increasingly large number of recombination events in each QTL interval are required to map the QTLs to decreasingly smaller intervals. Second, individual QTLs have small effects that are sensitive to the environment; there-

\section{SNP 1 QTL SNP 2 Strain EMO}

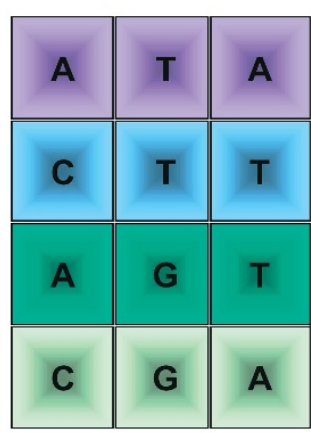

fore, the phenotype of a single individual is not a reliable indicator of QTL genotype. Third, even after whittling down the QTL interval to a region containing several positional candidate genes, one must determine which of them corresponds to the QTL. Guessing (the 'candidate gene' approach) has worked in cases where the genetic basis underlying the trait phenotype is well understood. But the availability of complete genome sequences for many organisms means it is possible to peruse the gene list in a candidate QTL region, inevitably finding many predicted 
a

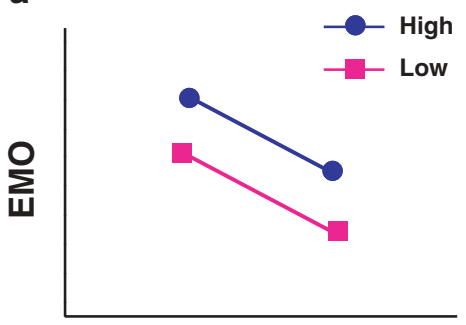

Mutant Wild-type b

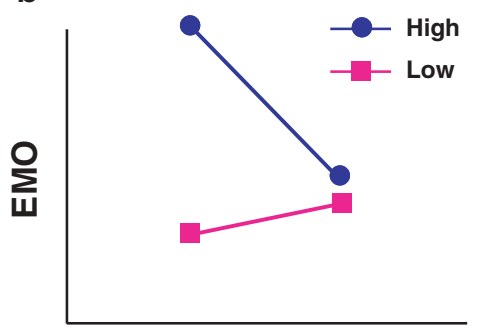

Mutant Wild-type

Figure 2 The quantitative complementation test. Anxiety-like behavior is assessed by an index (EMO) combining ambulatory behavior and defecation in the open-field environment for four genotypes: m/high, m/low, +/high and +/low. (a) Quantitative complementation. There is a difference in mean performance of the two parental strains when crossed to both mutant and wild-type; and, if the mutation is not completely recessive, there is a difference between mutant and wild-type strains when crossed to both high and low strains. The magnitude of the difference in phenotype between the two parent strains is the same in the background of the mutation and the wild-type allele, indicating the mutated allele does not interact with the QTL alleles. (b) Quantitative failure to complement. Here the magnitude of the difference in phenotype between the two parent strains is greater in the background of the mutation than the background of the wild-type allele, indicative of an interaction between the mutated allele and the QTL alleles.

genes of unknown function and a handful of known genes with no a priori relationship to the trait. This is particularly true for variation in complex behaviors, which have been shown in Drosophila melanogaster to be attributable to subtle variants in pleiotropic genes ${ }^{6,7}$. Alternative strategies such as scanning genes in the region for potentially causal sequence polymorphisms or differences in transcript abundance are not optimal. Even if only two lines are used to map the QTLs, there will be many sequence differences between them, and all will be associated with variation in the trait. It is not yet certain to what extent differences in transcript abundance will correlate with differences in gene expression at the level of trait phenotype, and small differences in expression confined to a few cells or tissues may be relevant but not detectable with current technology.

\section{Historical haplotypes}

Anxiety-like behavior is a classic quantitative trait in mice. When placed in a brightly lit open-field arena, reactive or anxious mice will freeze in place and defecate, whereas nonreactive mice will explore their environment and defecate less. Thus, measures of locomotion and numbers of fecal pellets in the open-field environment are two negatively genetically correlated traits that can be combined into a single index of 'emotionality', $\mathrm{EMO}^{8}$.

Previously, a QTL that affects EMO was mapped to a $0.8-\mathrm{cM}$ region on chromosome 1 , using a heterogeneous population of mice

derived from a cross of eight standard inbred lines ${ }^{8}$. Yalcin et al. ${ }^{5}$ argued that the increased historical recombination in an outbred population would enable even finer mapping of this QTL. They scored anxiety phenotypes for 729 individuals from the commercially available MF1 outbred strain and determined single-nucleotide polymorphism (SNP) genotypes for 42 markers spanning the 3.5$\mathrm{Mb}$ region to which the QTL mapped. Tests for association of each SNP genotype with variation in anxiety identified a single weakly significant marker, after adjusting the experiment-wide significance level to account for multiple tests. But consideration of associations of inferred progenitor haplotypes with differences in trait phenotypes can be more powerful than single-marker associations ${ }^{9}$ (Fig. 1). This multipoint mapping method was applied to the haplotypes of all 729 individuals, inferred by considering them a finegrained mosaic of either four or eight inbred strains. The single QTL fractioned into three closely linked QTLs, each of which contributed $\sim 5 \%$ of the total phenotypic variance and which did not interact epistatically. The first and third QTLs contained no known genes. The 95\% confidence interval for the second QTL contained only two genes, regulator of G-protein signaling 2 (Rgs2) and Rgs13.

\section{An anxious gene}

Quantitative complementation tests to mutations at positional candidate genes have been used in D. melanogaster to identify genes that functionally interact with, and possibly correspond to, the QTL alleles ${ }^{3}$. The test requires strains containing a mutated $(\mathrm{m})$ and a wild-type $(+)$ allele of the candidate gene, preferably in the same coisogenic background. These strains are then crossed to the strains containing the alternative QTL alleles (high or low) and the four $\mathrm{F}_{1}$ progeny genotypes (high/m, low/m, high/+, low/+) are scored for the trait. Quantitative failure of the mutation to complement the QTL alleles is inferred if the difference in mean phenotype between the high/m and low $/ \mathrm{m}$ genotypes is greater than that between the high/+ and low/+ genotypes (Fig. 2). This is detected as a statistical 'Cross' ( $\mathrm{m}$ or + ) by 'Line' (high or low) interaction in a two-way analysis of variance.

In the first application of this method to mammals, Yalcin et al. ${ }^{5}$ showed that a recessive knockout mutation of $\mathrm{Rgs} 2$ failed to complement the parental high- and low-anxiety QTL alleles. Rgs2 is widely expressed in the brain, and individuals homozygous with respect to the mutation are more anxious than wild-type mice. Failure to complement, whether qualitative or quantitative, can arise from an allelic interaction between the mutation at the candidate gene and the homologous QTL alleles, or from an epistatic interaction between the candidate gene mutation and nonhomologous segregating QTLs. In either case, however, Rgs2 is implicated as modulating natural variation in anxiety.

\section{From QTLs to genes}

Because the outbred strain of mice used in this study captures much of the allelic diversity among common inbred strains and is commercially available, it should be possible to use the method of probabilistic ancestral haplotype reconstruction to map QTLs affecting any quantitative trait in mice with high resolution. Such studies might show that single QTLs fractionate into multiple linked QTLs, as is common in D. melanogaster ${ }^{10,11}$. Finally, despite the emerging complexity of the genetic architecture of quantitative traits, it is possible to use quantitative complementation tests to identify genes that functionally interact with QTLs and thus modulate variation in the trait. The complementary efforts to generate mutations in all mouse genes will be an invaluable resource for systematically testing all positional candidate genes for interactions with QTLs, not just the few for which mutations are currently available ${ }^{12,13}$. This strategy is not restricted to mice but can be applied to any organism with the requisite genetic and genomic resources. This bodes 
well for eventually describing quantitative genetic variation in terms of complex genetics rather than complex statistics.

1. Falconer, D.S. \& Mackay, T.F.C. Introduction to Quantitative Genetics (Addison Wesley Longman, Harlow, Essex, 1996).

2. Lynch, M. \& Walsh, B. Genetics and Analysis of Quantitative Traits (Sinauer, Sunderland,
Massachusetts, 1998)

3. Mackay, T.F.C. Annu. Rev. Genet. 35, 303-339 (2001)

4. Glazier, A.M., Nadeau, J. \& Altman, T.J. Science 298, 2345-2349 (2002).

5. Yalcin, B. et al. Nat. Genet. 36,1197-1202 (2004)

6. Sokolowski, M. Nat. Rev. Genet. 2, 879-890 (2001)

7. Anholt, R.R.H. et al. Nat. Genet. 35, 180-184 (2003).

8. Talbot, C.J., et al. Nat. Genet. 21, 305-308 (1999).
9. Mott, R., Talbot, C.J., Turri, M.G., Collins, A.C. \& Flint, J. Proc. Natl. Acad. Sci. USA 97, 12649-12654 (2000).

10. Pasyukova, E.G., Vieira, C. \& Mackay, T.F.C. Genetics 156, 1129-1146 (2000).

11. Harbison, S.T., Yamamoto, A.H., Fanara, J.J., Norga, K.K. \& Mackay, T.F.C. Genetics 166, 1807-1823 (2004).

12. Austin, C.P. et al. Nat. Genet. 36, 921-924 (2004).

13. Auwerx, J. et al. Nat. Genet. 36, 925-927 (2004).

\title{
Gangliosides help stabilize the brain
}

\author{
Richard L Proia \\ Defects in the breakdown of gangliosides are associated with a class of disorders known as lysosomal storage \\ diseases. Now, a defect in the synthesis of gangliosides, glycolipids that contain sialic acid and are abundant in the \\ brain, has been shown to underlie an inherited form of epilepsy.
}

Epilepsy is a serious neurological disorder affecting as many as 60 million people worldwide ${ }^{1,2}$. Individuals with epilepsy suffer from repeated, spontaneous seizures due to a dysregulation of neuronal excitability. There is a genetic basis for $\sim 40 \%$ of cases, which are divided into symptomatic epilepsies (where there is a structural or metabolic abnormality of the brain) and idiopathic epilepsies (where there is no such abnormality). Most genetic forms of epilepsy are complex disorders, in which multiple genes, in combination with environmental factors, contribute to the disease. A small fraction of these disorders are caused by defects in a single gene. These mendelian epilepsies are particularly informative because the genes that underlie them can uncover mechanisms regulating neuronal hyperexcitability and because these genes may also contribute to the much larger group of nonmendelian epilepsies.

Idiopathic epilepsies have been linked to genes controlling ion channel function and receptor signaling on neurons, whereas symptomatic epilepsies have been linked to genetic defects causing neurodegeneration and disordered brain development. Now, on page 1225 of this issue, Simpson et al. ${ }^{3}$ describe a new gene underlying a severe early-onset symptomatic epilepsy syndrome. The gene, SIAT9, encodes a glycosyltransferase involved in the

Richard L. Proia is in the Genetics of Development and Disease Branch, National Institute of Diabetes and Digestive and Kidney Diseases, National Institutes of Health, Building 10, Room 9N-314, 10 Center DR MSC 1821, Bethesda, Maryland 20892, USA.

e-mail:proia@nih.gov synthesis of gangliosides, glycosphingolipids containing sialic acid that are abundant in the plasma membrane of neurons. Numerous genetic diseases have been linked to defects in the ganglioside degradation pathway ${ }^{4}$, but this is the first report of a human disease caused by a defect in ganglioside biosynthesis.

Simpson et al. ${ }^{3}$ began their work by searching for the genetic defect underlying an autosomal recessive epilepsy syndrome present in an Old Order Amish pedigree. The affected children in this pedigree develop seizure activity, including generalized tonic-clonic seizures, within the first year of life. Coincident with the onset of seizure activity, the affected children show stagnation in the acquisition of developmental milestones and subsequently undergo neurological decline and become blind.

Based on the assumption that the mutated gene derived from a single founder in this genetic isolate, the authors used homozygosity mapping to pinpoint chromosomal regions that might contain the gene of interest. They found a single region of homozygosity on chromosome 2p12-p11.2 and then looked for mutations among the $\sim 50$ candidate genes present in this interval. They discovered that SIAT9, encoding GM3 synthase, contained a nonsense mutation that was predicted to cause premature termination of the protein product. Analysis of plasma glycosphingolipids in affected individuals confirmed the predicted block in the ganglioside biosynthesis pathway, as marked by substantial changes in the levels of several key gangliosides ${ }^{3}$.

Gangliosides are amphipathic molecules composed of a ceramide lipid anchor attached to an externally oriented oligosac- charide chain of variable length and complexity ${ }^{5}$. They are found on the surface of essentially all mammalian cells but are particularly abundant on neuronal cell surfaces. These lipids are the primary glycoconjugates on neurons and carry most of the sialic acid present in the brain 6 . The oligosaccharide chains of the main brain gangliosides are assembled in a combinatorial fashion by a biosynthetic pathway controlled by a few key glycosyltransfereases (Fig. 1). GM3 synthase occurs at a pivotal point in this pathway, transferring a sialic acid residue to lactosylceramide to produce a simple ganglioside, GM3, which serves as a precursor to complex gangliosides of the a- and b-series that are common in the brain (Fig. 1). Thus, a deficiency of GM3 synthase results not only in the absence of GM3 ganglioside but also in the absence of many key gangliosides normally found in the brain.

\section{Lessons from knockout mice}

Because brain samples from the affected individuals described by Simpson et al. were not available for analysis of brain gangliosides and pathology, mice deficient in ganglioside glycosyltransferases are important for gaining insight into the potential mechanisms underlying this disorder. As predicted from the ganglioside synthesis pathway, mice with mutations in Siat9 divert the synthesis of complex brain gangliosides of the a- and b-series to gangliosides of the o-series. Unlike the individuals described by Simpson et al., however, these mice do not show obvious seizure activity or shortened lifespan ${ }^{7}$. The reason for the lack of an obvious seizure phenotype in Siat9 knockout mice is not known, but it could be due to differences between mice and humans 